\title{
A short history of space VLBI
}

\author{
Richard Schilizzi \\ University of Manchester \\ Manchester, UK \\ E-mail: richard.schilizziemanchester.ac.uk
}

Space VLBI has a long and rich history involving many innovative ideas, studies, cultural exchanges, disappointments and successes. This short paper cannot do justice to all parts of the story, and will pick out a few highlights from my perspective. The story is mainly that of the lead up to the two successful missions, VSOP-HALCA and RadioAstron, and will cover the three main stages of space VLBI: the early days from 1977 to 1982, the study stage from 1983 to 1988 , and the mission stage from 1989 to the present day. 


\section{Introduction}

Very long baseline interferometry (VLBI) at radio wavelengths provides us with the highest angular resolution of any form of astronomy and gives us images and physical information on very small scales in stars and galaxies. It did not take long after the first successful detection of fringes with independent stations on baselines of 100s of $\mathrm{km}$ in $1967[1,2]$ for attempts to be made with interferometer elements on inter-continental baselines [3]. Even in the pre-VLBI era, thoughts had already turned to space-based interferometers to surpass the restrictions posed by the size of the Earth (see Prologue), and less than a decade after first fringes, serious studies of space-ground interferometers as an intermediate step from purely ground-based to purely spacebased were being made.

More than twenty space VLBI missions have been proposed over the succeeding years (Gurvits, private communication), but only two have made it all the way to the launch pad and successful operation, VSOP-HALCA and RadioAstron. (VSOP stands for VLBI Space Observatory Program.) The success of these missions owes a great deal to the vision and persistence of their leaders - Hisashi Hirabayashi, Masaki Morimoto, and Minoru Oda (the "Three Samurai") for VSOP-HALCA and Nikolay Kardashev (the "Tzar") for RadioAstron. This is not only a tale of these two missions but what went before, and in between. It was not a linear process.
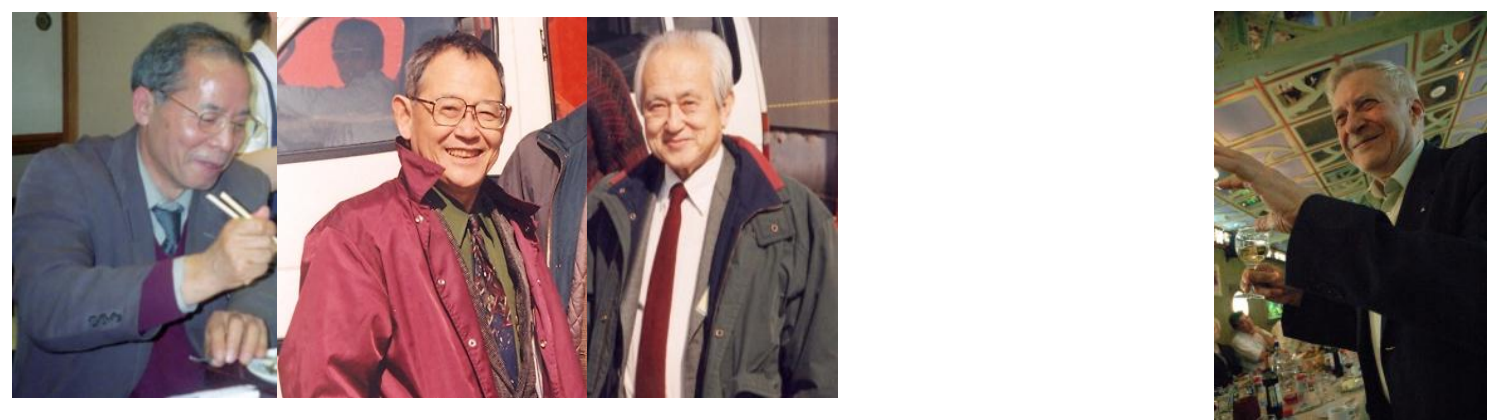

Figure 1. (left) The "Three Samurai", Hisashi Hirabayashi, Masaki Morimoto, and Minoru Oda, who were the prime movers behind VSOP-HALCA. (right) The "Tzar", Nikolay Kardashev, who was, and still is, the prime mover behind RadioAstron..

\section{Prologue}

In 1965, a paper on radiointerferometry with long baselines was published in Russian in the Soviet journal, Radiophysics, by L. I. Matveenko, N. S. Kardashev, and G. B. Sholomitsky (see Fig.2) . This set out how interferometers with independent elements might work. The original mauscript included a speculation about a purely space-based interferometer to escape the baseline length restrictions imposed by the Earth, but this section was removed from the published version to as discussed by Leonid Gurvits in these Proceedings. No mention was made of space-to-ground interferometry as an intermediate step, the step that has, in practice, been taken. 


\section{ON RADIOINTERFEROMETER WITH A LARGE BASE}

\section{I. Matveenko, N. S. Kardashev, G. B. Sholomitsky}

The radiointerferometer system without retranslation has been considered. The signal registration at the intermediate frequency is carried out independently by each antenna (by recording on a magnetic tape) with subsequent common processing of these results. The usage of two independent heterodynes imposes the following condition for their frequency stability $\sqrt{\overline{\Delta f_{\mathrm{h}}^{2}}} / f_{\mathrm{h}} \leqslant 1.6 \cdot 10^{-11} D(D$ is the base length in $\mathrm{km})$. The merits of such interferometer have been discussed.

Figure 2. Translation of the title and abstract of the 1965 paper by L. I. Matveenko, N. S. Kardashev and G. B. Sholomitsky. The original mauscript included a speculation about a purely space-based interferometer, but this was not included in the final version.

\section{Stage 1 - The early days of space VLBI: 1977-1982}

\subsection{USA}

US astronomers, Bob Preston and Bernard Burke, can justly be called two of the three fathers of space-ground interferometry, or space-VLBI as we now know it. The other "father" is Nikolay Kardashev. JPL Engineering Memorandum \#315-16 by Preston, dated 2 November 1977, explored how "satellite-borne VLBI terminals could be used to provide maps of compact radio sources with finer resolution, less ambiguity and more efficiency than earth-bound VLBI techniques." The aim was to show that there were sound scientific reasons for putting a large antenna in relatively low orbit earth orbit in addition to other communications goals.

Following up on this, Burke, Preston and colleagues (Tom Clark, Marshall Cohen, Ken Johnston, Ken Kellermann, Jim Moran, Alan Rogers, and Irwin Shapiro) submitted a proposal (AO-OSS-2-78) to NASA on 15 November 1978 called "Investigation and Technical Plan for a VLBI station on the 1981-1983 SpaceLab mission”. This proposal was successful, but NASA stopped its involvement in SpaceLab shortly thereafter. The next initiative was to deploy a parabolic antenna in earth orbit, this time attached to the shuttle (see Figure 3). The final report on the Mission Definition Study for a VLBI station utilizing the Space Shuttle (NAS-5-2554) was submitted by Burke and collaborators on 12 October 1982.

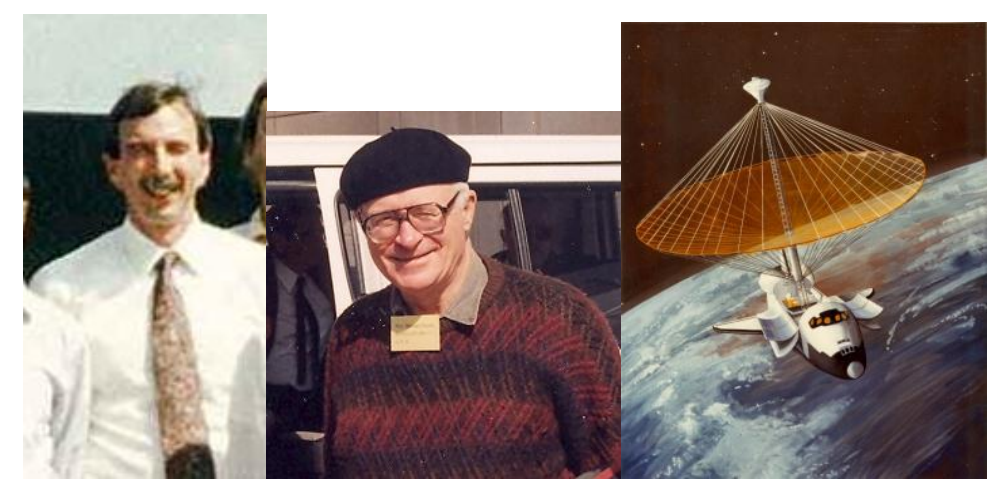

Figure 3. left Bob Preston, middle Bernie Burke, right an artist's conception of a 50m antenna attached to the space shuttle

About six weeks before submission of the final report in early September, Burke, Preston and Frank Jordan (JPL) participated in a conference on VLBI techniques in Toulouse, 
France, and there met a number of European radio astronomers in an effort to seek international support for the Shuttle mission concept. From the results of that meeting came QUASAT, which we will look at briefly in Stage 2 of this story.

\subsection{An important side-show: satellite-linked VLBI in Canada-USA and Europe}

By the mid-70s, the pioneers of VLBI and several generations of students and postdocs had learnt the hard way about the vicissitudes of Mark I and Mark II tape-recording VLBI, and the multitude of problems experienced by tape recorders operating at their limits. The attractions of using a geostationary satellite as a communications channel for VLBI data were obvious, not only operationally but as a means of increasing the sensitivity of the measurements through the use of a wider observational bandwidth. In 1977, Allen Yen, Ken Kellermann, Benno Rayhrer, Steve Knowles and their collaborators [4] pioneered the use of the Hermes communications satellite, a joint Canadian-US program, for VLBI between Algonquin Park and Green Bank. Although successful, these experiments were not pursued due to the expected high cost of commercial bandwidth for an operational system. Other experiments on phase transfer via another geostationary satellite, ANIK-B, continued for a few more years [5].

The idea was taken up by European scientists including Roy Booth, James Campbell, Ken Kellermann and myself, and an approach made to ESA in 1977 via Giancarlo Setti, at that time Chairman of the ESA Astronomy Working Group. A Mission Definition Study [6] was followed by a Phase A Study using L-SAT [7], and a series of experiments in phase transfer designed and led by Arnold van Ardenne using the European Orbiting Test Satellite [8]. The results of the studies and the phase transfer measurements led ESA to decide that L-SAT could be used for VLBI provided that the European VLBI stations paid for the ground stations. This proved too high a financial hurdle, and the idea quietly faded away, helped by the promise of the wide bandwidth Mark III recording system being developed at Haystack Observatory.

The one lasting positive effect of the involvement in satellite-linked VLBI in Europe was the change in visibility for radio astronomy in ESA from zero to high; our good connections in the agency proved invaluable when the Americans came calling in Sept1982.

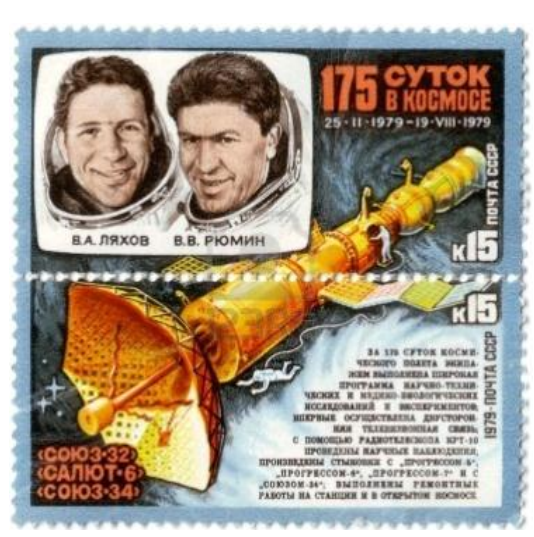

\subsection{Soviet Union}

Not surprisingly, given the early thoughts about space-based interferometers noted in the Prologue, Nikolay Kardashev and his colleagues developed their own program to deploy parabolic antennas on satellites for VLBI. In 1979, a 10m dish (KRT-10) was deployed on the Salyut-6 orbital station and carried out experiments in June - August. This was celebrated in the commemorative stamp shown in Figure 4. As far as radio astronomy was concerned, no useful results were obtained, but there is reason to believe that Earth-oriented measurements by other stakeholders may have been successful.

Figure 4. Soviet Union stamp commemorating the successful deployment of a $10 \mathrm{~m}$ diameter dish, KRT-10 in space in 1979. 


\subsection{Japan}

Similar discussions were taking place in Japan in 1981 and 1982, resulting in a paper by Hirabayashi et al in the Space Station Symposium in Tokyo in October 1982 proposing a large diameter radio astronomy dish on Space Station for VLBI with ground-based telescopes. This was the first contact between radio astronomers and ISAS, the Japanese Institute for Space and Aeronautical Science, and was important in engaging the interest and support of Minoru Oda, then ISAS Director-General, when proposals for a Japanese free-flyer on an ISAS rocket were made a few years later.

\section{Stage 2 - QUASAT, TDRSS, VSOP, and RadioAstron: 1983-1988}

Discussions over coffee in the main square in Toulouse on 1 September 1982 brought the US and European interests described in sections 3.1 and 3.2 together. There was rapid agreement on a free-flying scientific satellite as the most exciting concept. Fortuitously, a call for mission proposals was imminent in ESA, and this provided a route forward in Europe An Assessment Study took place from 1983 to 1985 [9] on a 15m diameter inflatable antenna operating at the standard ground-based network frequencies of 22,5 and $1.6 \mathrm{GHz}$ in an orbit with an apogee height of $25000 \mathrm{~km}$. This was to provide an increase of more than three times in baseline length. From its inception, QUASAT was studied as a joint ESA/NASA project with three joint meetings in the study period to ensure close coordination of the effort.

A workshop on QUASAT in June 1984 in Gross Enzersdorf in Austria [10] turned out to be a major event for space VLBI. Apart from showcasing the science to be done with space VLBI and progress on technology development, the first seeds of global cooperation were sown. Papers on the Soviet and Japanese space VLBI concepts given by Roald Sagdeev and Masaki Morimoto, provided the first opportunity for most European and US scientists to hear of these project ideas. Sagdeev discussed two potential ground-space interferometer projects, one a loworbit space telescope with very complete coverage of uv-plane, the other a high orbit space telescope providing very good angular resolution. Morimoto described the work of a joint study group in the Institute for Space and Astronomical Science (ISAS), Radio Research Laboratory and Nobeyama Radio Observatory, to study the possibility of a small antenna in space to do VLBI experiments. One of the conclusions of the Workshop was that it was desirable to find an appropriate means to continue the discussion on these projects.

This conclusion was given added weight for the QUASAT team when it was realised that it was impossible to combine superb uv-coverage with a substantial jump in angular resolution compared to ground-based VLBI. So why not combine forces and simultaneously fly two satellites in complementary orbits, and achieve "perfect" uv coverage out to $60000 \mathrm{~km}$ ? The QUASAT-centred thinking focussed on QUASAT + RadioAstron or QUASAT + the Japanese satellite.

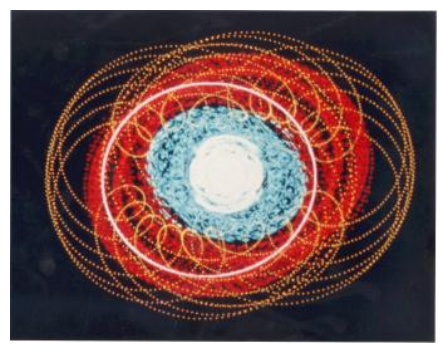

Figure 5. uv-plane coverage of a two-satellite space VLBI array. The central uv-plane coverage (white) comes from the groundbased array only, the blue coverage from the lower of the two satellites with the ground array, the red coverage from the higher satellite and the ground array, and the gold uv-tracks from the two space telescopes as a single interferometer. 
In March 1985, after considerable discussion complicated by political considerations related to US-Soviet relations, a COSPAR Ad-hoc Committee on Space VLBI was established under the chairmanship of Graham Smith to provide a forum for discussions on the ESA/NASA and Soviet efforts. In addition, the Inter-Agency Consultative Group (IACG, comprising NASA, ESA, Intercosmos, and ISAS) established a sub-Panel on Space VLBI under the chairmanship of Frank Jordan in July 1986 as a means of the space agencies informing themselves of developments. Both bodies were influential in keeping the profile of the various mission concepts relatively high amongst decision-makers. The meetings also served for the exchange of ideas on the space VLBI concepts so that dual satellite missions might, in principle, be possible. In the end nothing came of the dual satellite idea, but individually both VSOP-HALCA and RadioAstron owed something of their design philosophy to the QUASAT legacy.

In the early 1980s, RadioAstron was approved for launch as a 10m diameter telescope operating at four frequencies, 0.3, 1.6, 5 and $22 \mathrm{GHz}$ in an orbit with an apogee height of 100 $000 \mathrm{~km}$. It was one of three competing Spectrum missions, the others being Spektr X-Gamma and Spektr-UV. The hope was for the first launch in 1988. This proved to be somewhat optimistic but, interestingly, RadioAstron is the only one of the three to be launched so far. The international interest in the mission and the requirement for co-observing ground arrays meant there would have to be international involvement; this led to the establishment in October 1985 of an international Working Group on RadioAstron, the forerunner of the RadioAstron International Science Council (RISC).

Meanwhile, JPL, under the leadership of Gerry Levy, was spearheading a space VLBI demonstration using one of the Tracking and Data Relay System Satellites (TDRSS). The geostationary satellite carried two $4.9 \mathrm{~m}$ diameter dishes, one of which was used to observe the radio source and the other to transfer the data to a tracking station where it was recorded on a VLBI data acquisition unit for later correlation with the data from the ground array. This clever experiment was brilliantly successful with fringes in 1986 on 1730-130 (NRAO530), 1510-089 and 1741-038, detected at $2.3 \mathrm{GHz}$ on baselines up to 2.16 Earth diameters, and measured brightness temperatures above the canonical $10^{12} \mathrm{~K}$ limit [11].

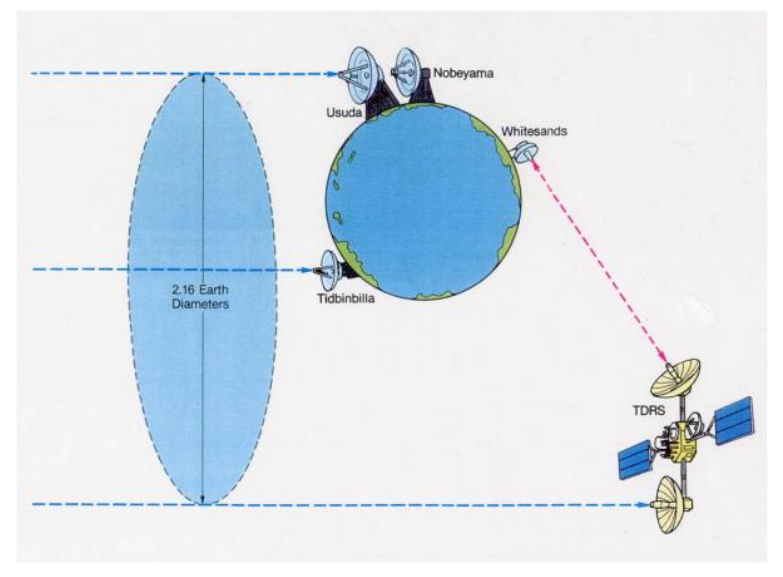

Figure 6. A schematic of the first space VLBI experiment in 1986 using one of the US Tracking and Data Relay System satellites to receive signals from the radio sources and then transmit them to a tracking station at Whitesands in the US for later correlation with the signals from the ground array (Tidbinbilla in Australia, Nobeyama and Usuda in Japan). 
QUASAT went into a Phase A study in ESA in February 1986 which lasted until June 1988. Team members were drawn from Europe, USA, Canada and Australia. The final report [12] was issued in time for the "shoot-out" in Oct 1988 to select which of several competing missions would go on to Phase C/D and launch. But QUASAT was disqualified two days before the presentation because it was judged to be too expensive as a stand-alone mission. There is much to be said about the process, but that will have to wait for another occasion. The eventual winner of this round was the Cassini-Huygens mission. Interestingly, ground-based VLBI tracking of the Huygens Probe actually saved an important Probe experiment when the nominal radio channel from Huygens to Cassini failed [13].

There were some last gasps by ESA and NASA in the area of space VLBI in the decade or so to come - International VLBI Satellite (IVS) studied by ESA and the Astro Space Center in Soviet Union/Russia, Advanced Radio Interferometry between Space and Earth (ARISE) studied by NASA, and Advanced Low Frequency Array (ALFA) again studied by NASA - to name three of the most prominent concepts. None made it past the feasibility stage.

\section{Stage 3 - VSOP-HALCA and RadioAstron: 1988-present day}

The great disappointment felt by the QUASAT team was tempered by the approval of VSOP for launch by ISAS a few months later, in December 1988. The concept was an $8 \mathrm{~m}$ diameter unfurlable antenna operating at 1.6, 5, and $22 \mathrm{GHz}$ in an orbit with an apogee height of 21600 $\mathrm{km}$. This was designated an engineering mission because, to add to the excitement, it was scheduled to be the payload, called Muses-B, on the first launch of the new ISAS M5 rocket.

Working closely with the Soviet Union on RadioAstron still did not have the seal of approval from some higher authorities...So the QUASAT team all took an oath of allegiance to the Three Samurai, while continuing to work with the Tzar, participating in advisory committee meetings and building receivers.

Papers by Hisashi Hirabayashi, Yuri Kovalev and Leonid Gurvits in these Proceedings describe the two mission concepts and their development from other perspectives and in more detail, so the remainder of this section will focus more on the international aspects of the missions.

\subsection{RISC and VISC}

The VLBI arrays in the US, Europe and Australia were key elements of both mission concepts and so International Science Councils were established with representation from all the stakeholders in order to review progress and provide advice on the space and ground segments. The ISCs met twice a year, starting in 1986 for the RISC and 1992 for the VISC.

The RISC was co-chaired by Nikolay Kardashev and a member of the international community on a two-year rotating basis, starting with Dave Jauncey. The adjustments that took place in post-Soviet Russia from 1992 affected the flow of funds to space science and this slowed progress on RadioAstron considerably. The RISC met regularly until September 1999 and then sporadically for the decade thereafter until the launch date firmed up. The decision to raise the RadioAstron apogee height from 100000 to the final value of $350000 \mathrm{~km}$ was taken by the Astro Space Center in the course of the 1990s. The VISC was initially co-chaired by Masaki Morimoto and Anton Zensus and continued to meet until 2006, well after the end of the mission in November 2005. 

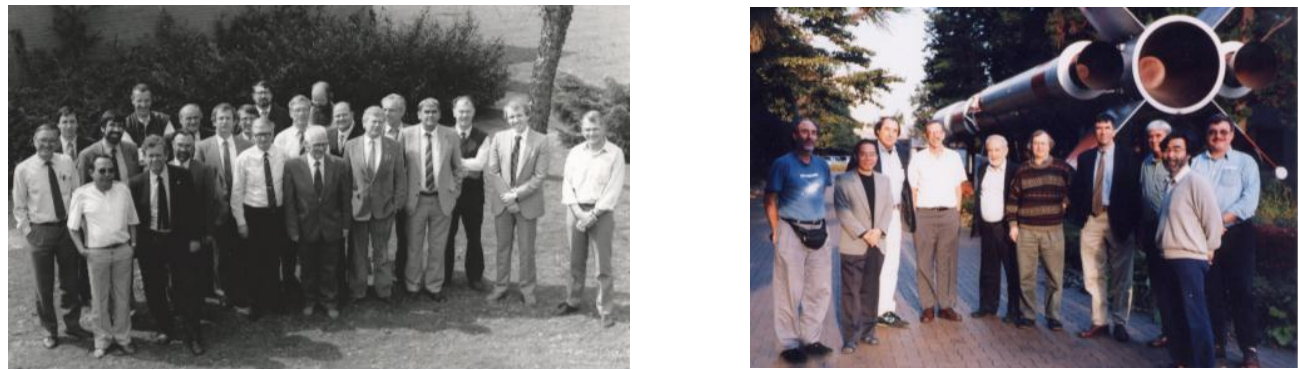

Figure 7. left: The $6^{\text {th }}$ RISC meeting in Dwingeloo, The Netherlands in April 1988. right: The first VISC meeting in Sagamihara, Japan in November 1992. There was considerable overlap in the membership which provided a level of continuity between the two missions.

In addition to the intensive engineering and science discussions, cultural exchanges formed an integral part of the ISC proceedings leading, in most cases, to greater mutual understanding.
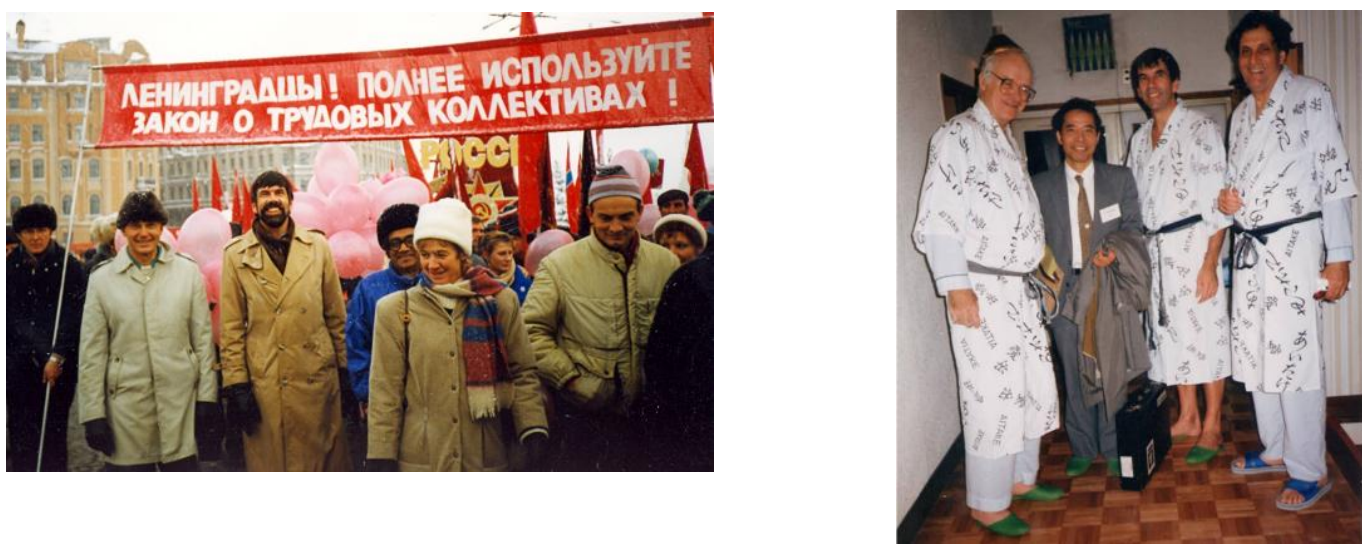

Figure 8. left: October Revolution Parade in Leningrad, November 1988. right: a chance meeting of Hirax Hirabayashi with Bernie Burke, Gerry Levy and the author in a Japanese ryokan.

During the late 1980s, a number of the IACG agencies encouraged, and provided support for, radio observatories to construct receivers for the orbiting telescope. In Europe, ESA provided test facilities for a $5 \mathrm{GHz}$ receiver designed and constructed by the Netherlands Foundation for Radio Astronomy and the Max-Planck Institute for Radioastronomy on behalf of the European VLBI Consortium, while the Finnish Space Agency supported a similar effort on the $22 \mathrm{GHz}$ receiver by Helsinki University of Technology working with NRAO, and CSIRO in Australia designed and constructed the $1.6 \mathrm{GHz}$ receiver. Similarly, a consortium of Indian organisations in collaboration with Russian institutes undertook the design and construction of the $327 \mathrm{MHz}$ receiver for RadioAstron. Flight models were delivered by the mid-1990s, but due to the delay in launch only the $1.6 \mathrm{GHz}$ receiver, of the original four delivered, actually went into space and successful operation.

\subsection{Global VLBI Working Group}

As preparations for VSOP began to intensify in 1993, it became clear that the ground arrays needed to plan their availability on a global scale to make optimum use of the space resource. Roy Booth, Ron Ekers and Paul Vanden Bout initiated the Global VLBI Working 
Group at a meeting in the Capitol bar in Socorro, New Mexico, USA in 1993. The prime aim of the GVWG was to serve as a liaison between ground-based observatories and national or international space agencies, for coordination of participation by ground radio telescopes in Space VLBI missions. In this, it was eminently successful. Agreements guaranteeing array availability for VSOP were made, and a joint VSOP Program Committee was established by the arrays to review and select observing proposals. Agreements were also made on the format and correlation of the data. Roy Booth chaired the GVWG throughout the pre-VSOP launch period.

\subsection{VSOP in orbit}

VSOP was launched on 12 February 1997, and re-named VSOP-HALCA. The collective excitement of the international partners assembled a few $\mathrm{km}$ from the launch site can be seen in Figure 9. A much fuller account of this magnificent achievement can be found in the contribution by Hirax Hirabayashi in these Proceedings.

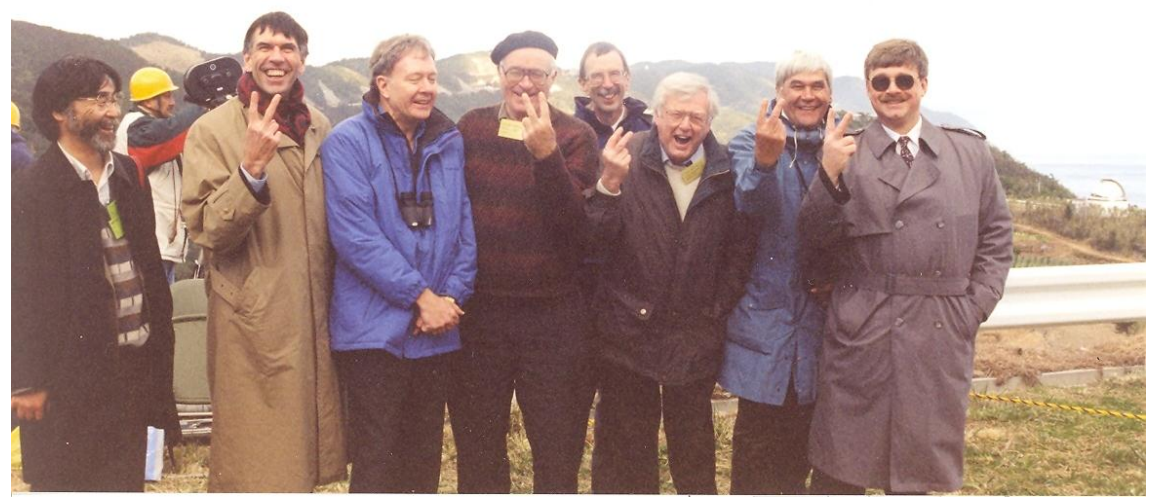

Figure 9. After the successful launch. "V" for victory and VSOP-HALCA

The VSOP-HALCA mission observed compact radio sources successfully at 5 and 1.6 $\mathrm{GHz}$ for more than 6 years [14], with one of the most interesting results from the increased angular resolution being the measurement of transverse structure in the $3 \mathrm{C} 273$ jet (Fig. 10).
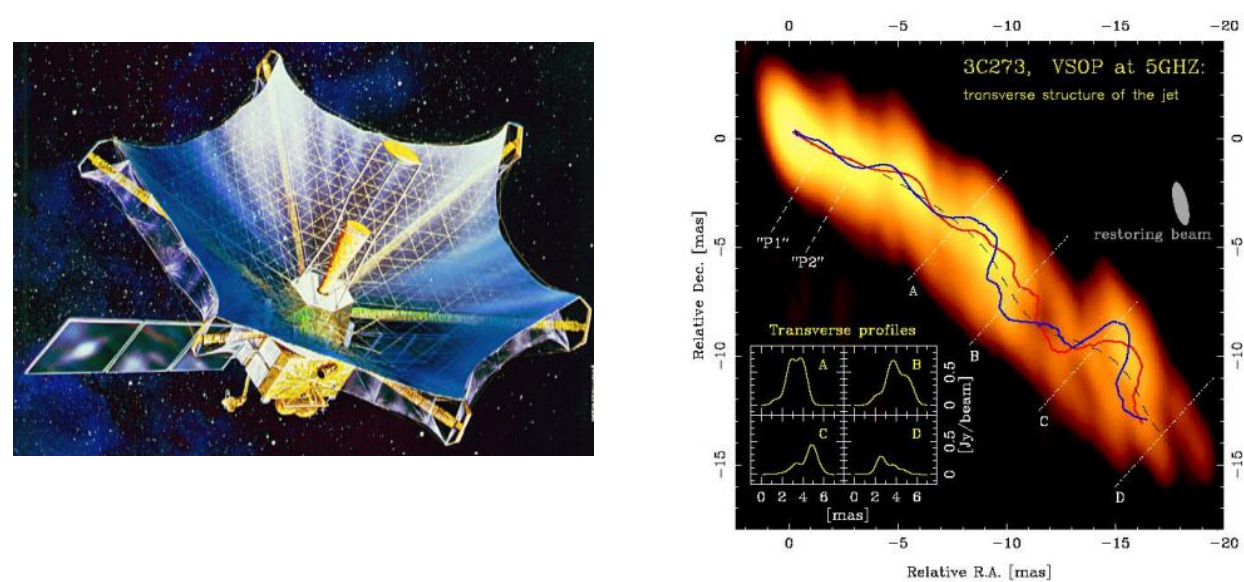

Figure 10. left: Artist's depiction of VSOP-HALCA in orbit. right: The jet in 3C 273 observed with VSOP-HALCA and the VLBA+Effelsberg [15]. Transverse structure is seen in the jet for the first time. 


\subsection{RadioAstron in orbit}

After more than 30 years in the making, Nikolay Kardashev and his team saw RadioAstron launch successfully from Baikonur on 18 July 2011, a superb achievement. After a commissioning period of several months during which first fringes were detected at a record physical baseline length (see Fig. 11), early science observations on active galactic nuclei, masers and pulsars are now taking place, and more detailed studies are being planned. Yuri Kovalev gives a much fuller account of the mission in his paper in these Proceedings.

Brightness temperatures of up to $10^{15} \mathrm{~K}$ in celestial radio sources can be sampled with the RadioAstron baselines. If such sources exist in AGN, our understanding of the physics of radio emission close to the central black holes in massive galaxies will be challenged.

It is true to say that RadioAstron has a bright future!

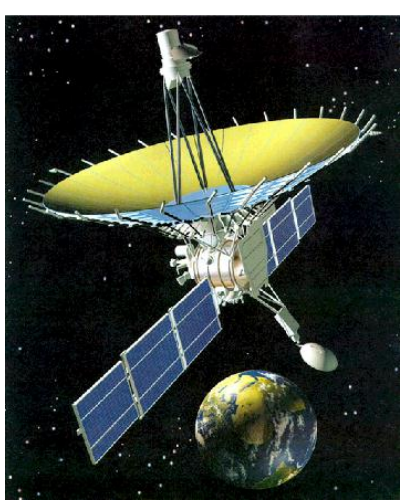

RADIOASTRON

$100000 \mathrm{~km}$ from Earth

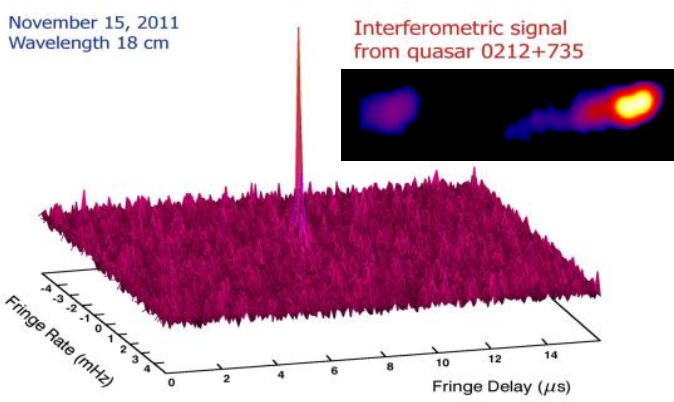

Figure 11. left: An artist's impression of RadioAstron in orbit. right: First fringes with RadioAstron on the longest physical baseline ever achieved. The spacecraft was $100000 \mathrm{~km}$ from Earth, observing 0121+735 at 1.6 GHz together with the Effelsberg telescope.

\section{Acknowledgements}

I have benefited greatly from discussions on ancient history with Leonid Gurvits, Bob Preston, Hirax Hirabayashi, David Jauncey and Ken Kellermann. They also dug deep in their archives for some of the figures included in this paper.

\section{References}

[1] N. W. Broten et al, 1967, Science 156, 1593

[2] C. Bare et al, 1967, Science 157, 189

[3] K. I. Kellermann et al, 1968, Astrophysical Journal 153, L29

[4] J. L. Yen et al, 1977, Science, 198, 289

[5] W. Cannon, 1983, in Proceedings of VLBI Techniques, CNES, p443

[6] R. T. Schilizzi et al, 1978, ESA Mission Definition Study of VLBI Using a Geostationary Satellite, DP PS (78)

[7] R. T. Schilizzi et al, 1981, ESA Phase A Study of VLBI using a Geostationary Satellite, $\operatorname{SCI}(80) 1$ 
[8] A. van Ardenne, J. D. O’Sullivan, 1983, in Proceedings of VLBI Techniques, CNES, p459

[9] R. T. Schilizzi et al 1985, ESA Assessment Study on QUASAT, a Space VLBI Satellite, SCI(85)5

[10] P. Burke, 1984, Editor, Proceedings of QUASAT, a VLBI Observatory in Space, ESA SP-213

[11] G. Levy et al, 1986, Science, 234, 187

[12] R. T. Schilizzi et al, 1988, ESA Phase A Study of QUASAT, a Space VLBI Satellite, SCI(88)4

[13] J.-P. Lebreton et al., 2005, Nature 438, 800

[14] H. Hirabayashi et al, 1998, Science, 281, 1825

[15] A. Lobanov, 2009, in Proceedings of Approaching Micro-Arcsecond Resolution with VSOP-2:

Astrophysics and Technology, p189 